\title{
Characterization of a Chaotic Telecommunication Laser for Different Fiber Cavity Lengths
}

\author{
Valerio Annovazzi-Lodi, Senior Member, IEEE, Sabina Merlo, Member, IEEE, Michele Norgia, Member, IEEE, and \\ Alessandro Scirè, Member, IEEE
}

Invited Paper

\begin{abstract}
In this paper, we consider a single-mode telecommunication laser emitting at $1550 \mathrm{~nm}$, which is subjected to backreflection from a mirror at the end of a long fiber cavity. In view of cryptographic applications, we focus our analysis on the chaotic regime, which we investigate as a function of pump current, backreflection level, and cavity length. Experimental data are compared with numerical simulations, showing the advantages of the fiber approach.
\end{abstract}

Index Terms-Chaos, cryptography, laser stability, nonlinear oscillators, optical communication.

\section{INTRODUCTION}

$\mathbf{T}$ HE STABILITY of semiconductor lasers subject to external injection and optical feedback has been analyzed by different authors in the last decade [1]-[4].

As is well known, for increasing levels of feedback or of injection, the laser undergoes a route from stability to coherence collapse, showing a rich variety of nonlinear dynamic regimes, such as undamped relaxation oscillation, chaos, bridges of periodic solutions [5], and low-frequency fluctuations [6], [7], where a global bifurcation leads the system into a highly nonlinear dynamics, involving wide orbits surrounding the external cavity modes (ECMs) ensemble.

Besides the theoretical interest for the different regimes that are observed, feedback phenomena are usually considered as a drawback, and substantial efforts have been made in order to produce lasers which are less sensitive to backreflection, or to develop pigtailing and packaging techniques which can reduce such effects.

In recent years, however, considerable interest has been attracted by optical cryptography. In this application, instead of trying to prevent the laser instability, one exploits the peculiar characteristics of the chaotic regime, where the laser shows a deterministic but very complicated modulation in the time domain, a large spectrum widening, and a strong dependence on

Manuscript received February 12, 2002; revised April 22, 2002. The work of A. Scirè was supported by a Marie Curie Individual Fellowship. This work was supported by the European Community under Contract ST 200029683 (OCCULT Project).

V. Annovazzi-Lodi, S. Merlo, and M. Norgia are with the Dipartimento di Elettronica, Universita' di Pavia, I-27100 Pavia, Italy.

A. Scirè is with the Instituto Mediterraneo de Estudios Avanzados (CSICUIB), E-07071 Palma de Mallorca, Spain.

Publisher Item Identifier 10.1109/JQE.2002.801948.

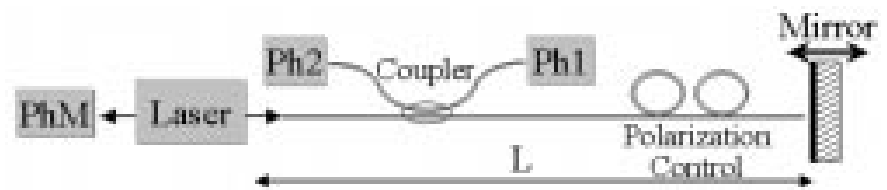

Fig. 1. Experimental setup for the stability measurements on the chaotic laser.

small variations of its parameters and on the starting conditions. Indeed, it has been shown that the chaotic regime can be used to hide information [8]-[12], either by mere superposition (masking), or by encoding a digital message on different chaotic attractors or orbits (e.g., by a chaos shift keying (CSK) scheme). Decoding is then based on the synchronization of two similar chaotic lasers in a master-slave arrangement.

In this work, we report on the characterization of a telecommunications laser subject to backreflection from a mirror at the end of a fiber cavity. This analysis is important in view of cryptographic applications, since a fiber implementation should provide an easy way to work with a long cavity to increase the chaos dimension [7], [13] and should be easy to insert in a telecommunications link. The experimental results will be compared with numerical simulations.

\section{EXPERIMENTAL SETUP}

Our experimental setup is shown in Fig. 1. The laser is driven to chaos by backreflection from a mirror [1]-[3], [14]; this scheme has been selected since it has the advantage of being all-optical, which implies that its speed is not limited by electrooptical conversion. Also, as compared to injection from another source [4], this approach is less expensive and its experimental implementation is easier. A fiber external cavity has been used, as a first step, to implement an all-fiber chaotic transceiver. With this aim, a laser in the third window has been used, since this is one of the standard wavelengths for telecommunications networks. The laser output and the reflected power are measured by photodiodes $\mathrm{Ph} 1$ and $\mathrm{Ph} 2$. Photodiode $\mathrm{Ph} 1$ is a high-speed device and its output signal is also fed to an RF analyzer to observe the electrical modulation spectrum of the laser. The internal photodiode PhM was used only for monitoring.

The laser source was a distributed-feedback (DFB) singlemode laser emitting at $1551 \mathrm{~nm}$ (Mitsubishi ML 976H11F). Its 
threshold current was $I_{\mathrm{th}}=7.3 \mathrm{~mA}$ at $25^{\circ} \mathrm{C}$. The laser temperature was stabilized within $0.01{ }^{\circ} \mathrm{C}$ by a Peltier cell. The DFB technology has been preferred because, as reported in the literature [15], and confirmed by preliminary tests, such lasers can maintain single-mode operation even in chaotic conditions; moreover, their center wavelength does not change appreciably, and their linewidth, even broadened by chaos, remains contained within a single channel of the dense wavelength division multiplexing grid. On the other hand, Fabry- Perot lasers are cheaper, but it is very difficult, at least in the third window, to obtain single-mode operation with high extinction of the side modes in the chaotic regime.

The main parameter to be controlled in the experimental arrangement of Fig. 1 is the feedback parameter $K$, i.e., the fraction of the electric field amplitude of the laser output which is injected back into the source. Parameter $K$, which represents the order parameter of the transition to chaos [14], is modified by varying the attenuation along the path between the laser and the mirror.

In our experiments, $K$ was varied by translating and tilting the mirror. The measurement of $K$ was performed by determining the square root of the ratio of powers at photodiodes $\mathrm{Ph} 2$ and $\mathrm{Ph} 1$. This value was then corrected to take into account the coupler splitting ratio and the laser-to-fiber launch efficiency. Observation of $\mathrm{Ph} 2$ output alone would give significant errors especially at high $K$, because the laser power also depends on the back-injected power.

The setup was built by using single-mode standard telecommunication optical fiber which will be easy to connect in the future to a standard fiber-optic link.

Even for relatively long cavity lengths $(>3 \mathrm{~m})$, we avoided using a polarization maintaining fiber. The fiber cavity was simply positioned on a horizontal plane to form a large coil with a radius of about $30 \mathrm{~cm}$, while the laser emission was linearly polarized in the vertical direction. The relatively small rotation and ellipticity of the laser field due to propagation was thus measured at the mirror output, and then compensated by using two retarders consisting of small loops made on the fiber cavity and properly oriented [16].

At this stage, we used an external (bulk) mirror because this arrangement provides an accurate control of position, and thus of $K$, and because it allows us to check the output polarization at the mirror end.

However, after characterization, when the chaotic laser will be a part of a more complex setup, metal deposition on the fiber end will be preferable to a bulk mirror, to implement a more practical all-fiber system. Then, trimming of $K$ could be conveniently done by using an attenuator made of a small loop positioned at an arbitrary location along the fiber. Especially for long cavities, polarization control could be performed (as required in synchronization experiments) by monitoring the polarization state through the (partial) end mirror.

It must be observed that, with a long fiber, care must be taken also to avoid variations of the polarization state with time. For this reason, the fiber setup must be stable. This requirement gives a practical limit to the cavity length, which, in our experience, should not be longer than $10-15 \mathrm{~min}$ in a laboratory environment.
In our setup, laser-to-fiber coupling was made by using aspherical antireflection-coated lenses, obtaining an efficiency of about $40 \%$. Using a tapered fiber did not improve this figure significantly, and had the drawback of requiring the laser package to be opened.

All fibers were terminated by an angled cut $\left(10^{\circ}\right)$ to avoid unwanted reflections.

\section{THEORY AND SIMULATIONS}

A single-mode semiconductor laser subject to optical feedback can be described in terms of the well-known LangKobayashi model [1]

$$
\begin{aligned}
\frac{d E(t)}{d t}= & \frac{1}{2}(1+i \alpha)\left[G(N)-\frac{1}{\tau_{p}}\right] E(t) \\
& +\frac{K}{\tau_{\text {in }}} E(t-\tau) \exp (-i \Omega \tau) \\
\frac{d N(t)}{d t}= & R p-\frac{N(t)}{\tau_{s}}-G(N)|E(t)|^{2} \\
G(N)= & \xi\left(N(t)-N_{t}\right)
\end{aligned}
$$

where

$E(t)$ slowly varying amplitude of the electric field;

$N(t)$ carrier density;

$K \quad$ feedback parameter;

$\xi \quad$ modal gain;

$\Omega \quad$ solitary laser frequency;

$\alpha \quad$ linewidth enhancement factor;

$R p$ pump parameter;

$N_{t} \quad$ carrier density at transparency.

Parameters $\tau_{s}$ and $\tau_{p}$ are the carrier and the photon lifetime, $\tau_{\text {in }}$ is the time of flight in the laser, and $\tau=2 n_{c} L / c$ is the time of flight in the external cavity of refractive index $n_{c}$.

In order to simplify the calculations, (1) and (2) can be conveniently rewritten as follows [17]:

$$
\begin{aligned}
\frac{d \mathbf{E}\left(t^{\prime}\right)}{d t^{\prime}} & =(1+i \alpha) n\left(t^{\prime}\right) \mathbf{E}\left(t^{\prime}\right)+\eta \mathbf{E}\left(t^{\prime}-\tau\right) \exp (-i \Omega \tau) \\
T \frac{d n\left(t^{\prime}\right)}{d t^{\prime}} & =p-n\left(t^{\prime}\right)-\left(2 n\left(t^{\prime}\right)+1\right)\left|\mathbf{E}\left(t^{\prime}\right)\right|^{2}
\end{aligned}
$$

In these equations, $\eta=K\left(\tau_{p} / \tau_{\text {in }}\right), T=\tau_{s} / \tau_{p}$, while $n, \mathbf{E}$, and $p$ are the normalized carrier density, electric field, and pumping term, respectively [18], and time $t^{\prime}=t / \tau_{p}$ is also normalized for convenience. By writing the complex field as $\mathbf{E}\left(t^{\prime}\right)=A\left(t^{\prime}\right) \exp \left(i \phi\left(t^{\prime}\right)-i \Omega t^{\prime}\right)$, and introducing the phase delay $\Delta\left(t^{\prime}\right)=\phi\left(t^{\prime}\right)-\phi\left(t^{\prime}-\tau\right)$ corresponding to the round-trip time $\tau$, the stationary solutions are found [17], [19] as fixed points $\left[A_{s}, n_{s}, \Delta_{s}\right]$ in the phase space of the three variables $\left[A\left(t^{\prime}\right), n\left(t^{\prime}\right), \Delta\left(t^{\prime}\right)\right]$. For $A_{s}$ and $n_{s}$, explicit expressions are available as

$$
n_{s}=-\eta \cos \Delta_{s} \quad \text { and } \quad A_{s}^{2}=\frac{p-n_{s}}{1+2 n_{s}}
$$

while $\Delta_{s} \equiv \omega_{s} \tau$ are the solutions of the implicit equation

$$
\begin{aligned}
\Delta_{s}-\Omega \tau & =-\eta \tau\left(\alpha \cos \Delta_{s}+\sin \Delta_{s}\right) \\
& =-\eta \tau \sqrt{1+\alpha^{2}} \sin \left(\Delta_{s}+\operatorname{arctg} \alpha\right) .
\end{aligned}
$$




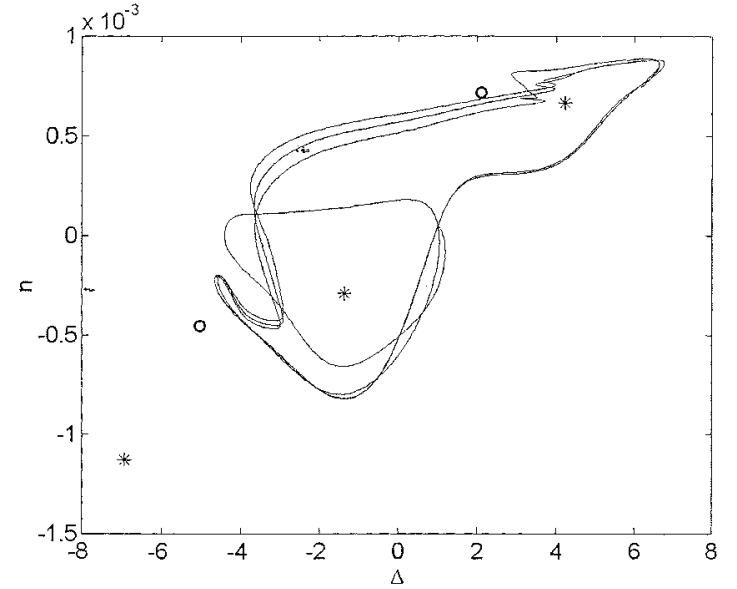

(a)

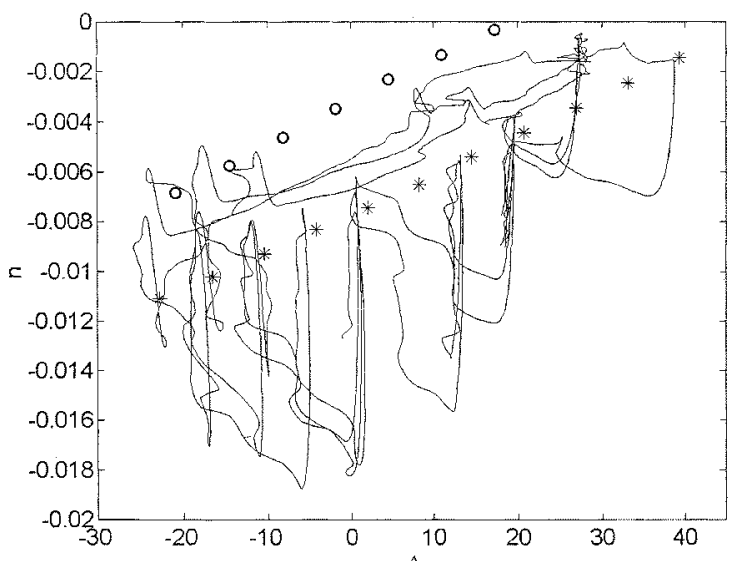

(b)

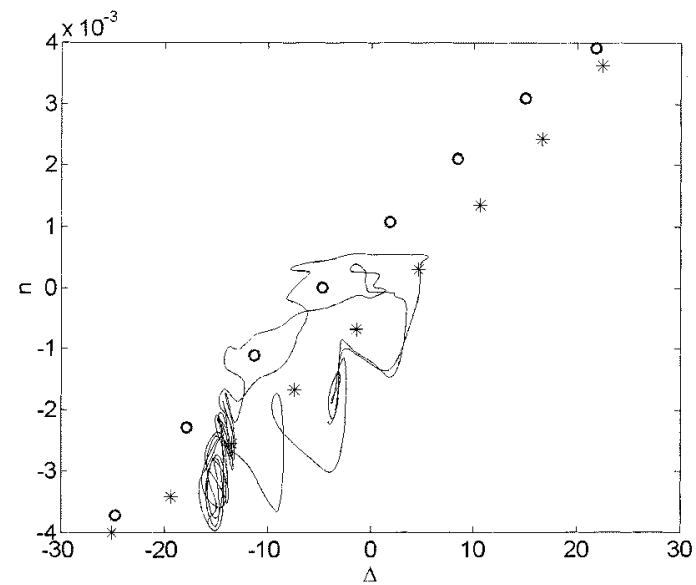

(c)

Fig. 2. Projection of the laser dynamics on the $[\Delta, n]$ plane for $\alpha=6, p=0.001, \tau=1000, \Omega \tau=-1$. Asterisks mark the stable fixed points (ECMs), open circles indicate the unstable fixed points (antimodes). Different regimes are shown. (a) Dynamics starts to experience the neighboring ECMs $\left(\eta=14.3410^{-4}\right.$ ). (b) Attractor crisis: the chaotic attractor is wandering through a wide range of ECMs $(\eta=0.01536)$. (c) Dynamics starts to contract $(\eta=0.998)$.

Equation (7) is the well-known Adler equation, which comes into play in many problems concerning forced lasers [2]. It has been shown [17], [19] that the fixed points lay upon an ellipse in the $(n, \Delta)$ plane. For low $\eta,(7)$ has only one solution, which represents the unperturbed laser mode. As $\eta$ increases, new fixed points are created in pairs, and for the different values of $\eta$, one point of each pair is unstable, while the stability of the other, which represents an ECM, depends on the effective feedback strength $C$

$$
C=\eta \tau \sqrt{1+\alpha^{2}}
$$

[see (7)] which, for a given cavity length, depends on $K$.

To study the system evolution, (4) and (5) must be numerically integrated in the general case. However, analytical results can be obtained under the approximation $A\left(t^{\prime}\right) \approx A\left(t^{\prime}-\tau\right)$, an assumption which is valid only for a relatively low feedback level $\left(K<510^{-3}\right)$ and for small $\tau$. The different regimes, that are observed at increasing feedback level, will be summarized in the following [13], [17], [20]. Some phase-plane plots are shown in Fig. 2(a)-(c) for different $\eta$, assuming for the other parameters the same values as in [17].

In the weak feedback regime $(K \ll 1)$, the laser oscillates near its unperturbed frequency $\Omega$. By increasing $K$, an ECM and an unstable fixed point are created. At higher feedback level, such an ECM becomes unstable, a first Hopf bifurcation takes place, and the laser exhibits undamped relaxation oscillations near the ECM. A limit cycle appears in the phase plane, which, by increasing feedback further, undergoes period doubling bifurcations, and is rapidly replaced by a chaotic attractor.

For larger $K$ values, this attractor grows in size, until the system trajectories begin to extend around the neighbor ECMs. Fig. 2(a) shows the first enlargement of the attracting region, for a value of $K$ where the laser dynamics involves two ECMs. Then, the system enters the so-called "chaotic attractor crisis" [17]. In this peculiar regime, the chaotic attractor is finally delocalized over a number of ECMs that increases with $K$ [Fig. 2(b)], until a maximum dimension chaos [13] is reached. Then, further increasing the feedback results in an inverse transition: in accord with experimental evidence, the dynamics starts to contract, the number of ECMs involved in the oscillation decreases [Fig. 2(c)], finally leading the laser again to a stable state, i.e., to work on a single narrow-linewidth mode. [20], [21]. As in unperturbed conditions, in this regime the laser output exhibits no RF modulation, which, instead, is observed in the more complex intermediate dynamics.

We remark that the analysis of the system stability under the assumption $A\left(t^{\prime}\right) \approx A\left(t^{\prime}-\tau\right)$, cannot include this inverse tran- 
sition, since it occurs at a relatively high feedback level. The same problem was found with the laser subject to external injection, which is described by a similar equation set. In spite of experimental evidence, the standard stability analysis failed to describe the inverse transition from chaos to stability, since amplitude fluctuations were neglected, and a better approximation was required [4].

The analysis of the inverse transition is an important theoretical issue and will be the subject of future investigation. For the moment, we have carried out numerical simulations on (4) and (5) to find the region of maximum dimension for the chaotic attractor, which is expected to represent the optimum operating point for cryptographic applications. Comparison of experimental and theoretical data will be presented in the following.

\section{EXPERIMENTAL MEASUREMENTS}

Our laser has been characterized in the chaotic regime with the setup of Fig. 1 for four different fiber lengths: $L=87.5 \mathrm{~cm}$, $1.45 \mathrm{~m}, 4.28 \mathrm{~m}$, and $9.375 \mathrm{~m}$. All of these cases fall into the so-called long-cavity regime [22], where phase effects are negligible, and in fact no regime variation has been observed upon small (less than $1 \mu \mathrm{m}$ ) mirror translations. Fiber implementation is well-suited for long cavities, since the fiber can be coiled to get a relatively compact setup. On the other hand, short cavities have been studied in the air [23], but they are difficult to implement in fiber, where the minimum length is practically limited by the coupler and by the launch optics.

The dynamic range of $K$, which could be achieved with our setup, spanned from $K=10^{-1}$ to $K=10^{-4}$. The maximum value was limited by the launch efficiency, the coupler splitting ratio, and the minimum attenuation at the mirror end. The minimum value corresponded to the unperturbed laser regime, and was limited by residual reflections of the fiber ends.

Before starting systematic measurements on the fiber cavity, the regimes which the laser undergoes have been quickly evaluated. For example, a sequence of spectra is reported in Fig. 3 for a laser driving current $I=10.3 \mathrm{~mA}, L=0.72 \mathrm{~m}$, and increasing $K$ values.

The spectra were measured by a RF spectrum analyzer fed by the output signal of PH1, which had a bandwidth of $6 \mathrm{GHz}$. For $K=0$ the laser is unperturbed and no modulation spectrum was detected. For increasing $K$, the relaxation frequency peak was experimentally observed: it corresponds to the limit cycle arising after the first Hopf bifurcation, which then grows into a low-dimensional chaotic attractor. Then other ECMs come into play, and thus more peaks arise [Fig. 3(a)]. The size of the chaotic attractor increases, until the spectrum becomes almost continuous and the dynamics is delocalized into a high-dimensional global chaotic attractor [Fig. 3(b) and (c)] involving a huge number of ECMs. This region lasts for a $K$ span which depends on the pump current, and represents the maximum dimensionality of the chaotic attractor, which can be observed at that external cavity length.

Then, the system begins the inverse transition: other less involved regimes follow for larger $K$, which are characterized by large peaks, but no longer by a continuous spectrum [Fig. 3(d)]. Finally, the laser reaches stability again. (a)

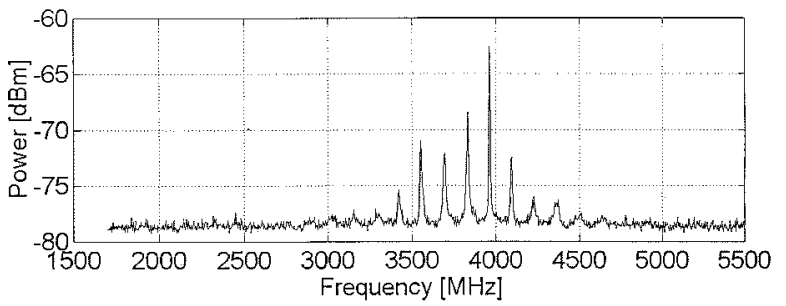

(b)

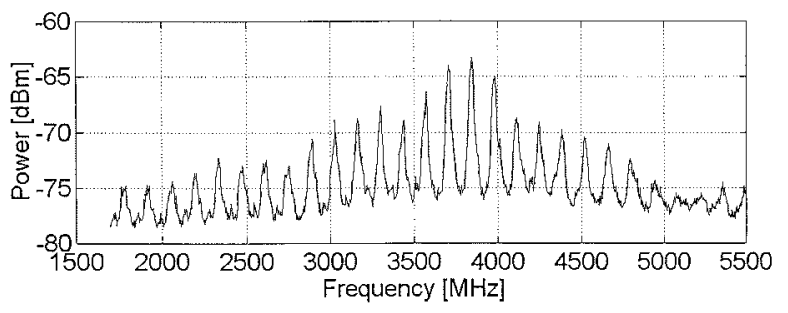

(c)

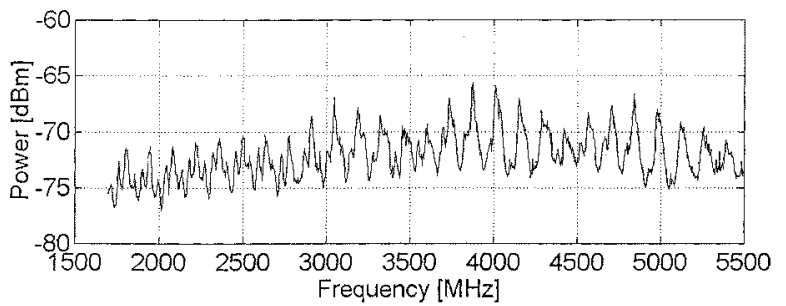

d)

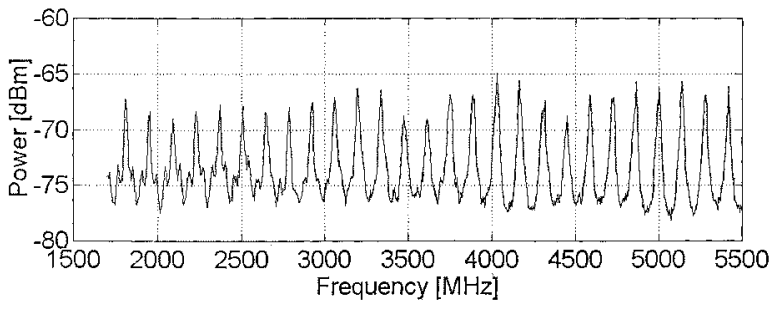

Fig. 3. Experimental spectra at constant current for different values of $K(L=$ $0.72 \mathrm{~m})$.

During all its route through the different regimes, the optical spectrum of the laser, measured by an optical spectrum analyzer, was always found to be confined within the instrument resolution $(0.2 \mathrm{~nm})$. The maximum threshold reduction was about $0.5 \mathrm{~mA}$.

From these preliminary observations, the regime of Fig. 3(b) and (c) was selected as promising for further investigations, since it is characterized by a relatively large continuous spectrum, and its frequency span and its amplitude seems adequate to hide a signal.

To compare numerical and experimental data at different cavity lengths in view of cryptographic applications, we have arbitrarily defined a boundary for the "maximum dimension chaos," by assuming the amplitude of the continuous spectrum to be at least $2 \mathrm{~dB}$ over the noise baseline measured in unperturbed conditions.

In Fig. 4(a) and (b), we have reported the boundaries of our "maximum dimension chaos" for the four different fiber lengths. For the sake of clarity, we have not superposed all theoretical and experimental data onto a single figure, but we have preferred to plot all experimental data in Fig. 4(a) and all theoretical curves in Fig. 4(b). However, to allow for a comparison, we have also shown the theoretical curve for $L=9.375 \mathrm{~m}$ in Fig. 4(a) and the experimental data for the same length in Fig. 4(b). 


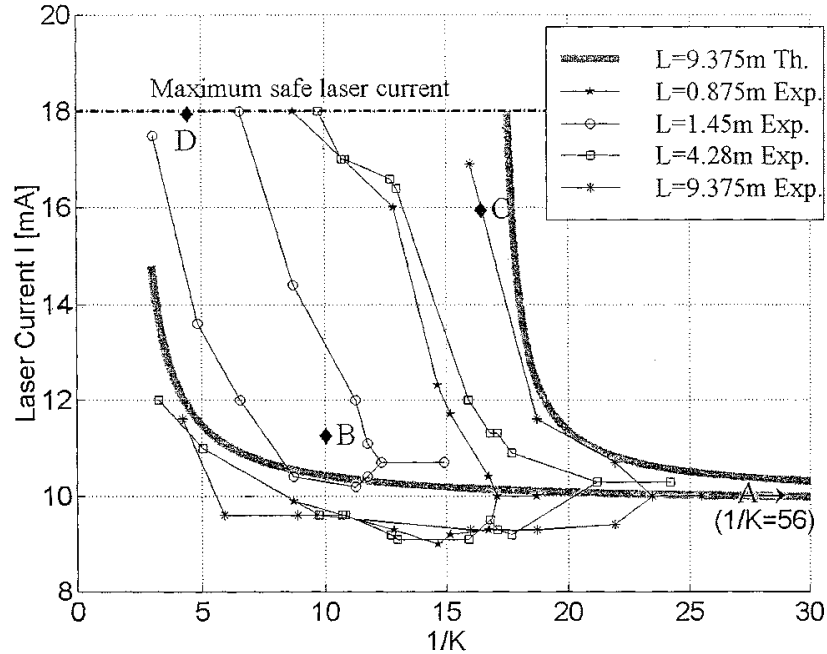

(a)

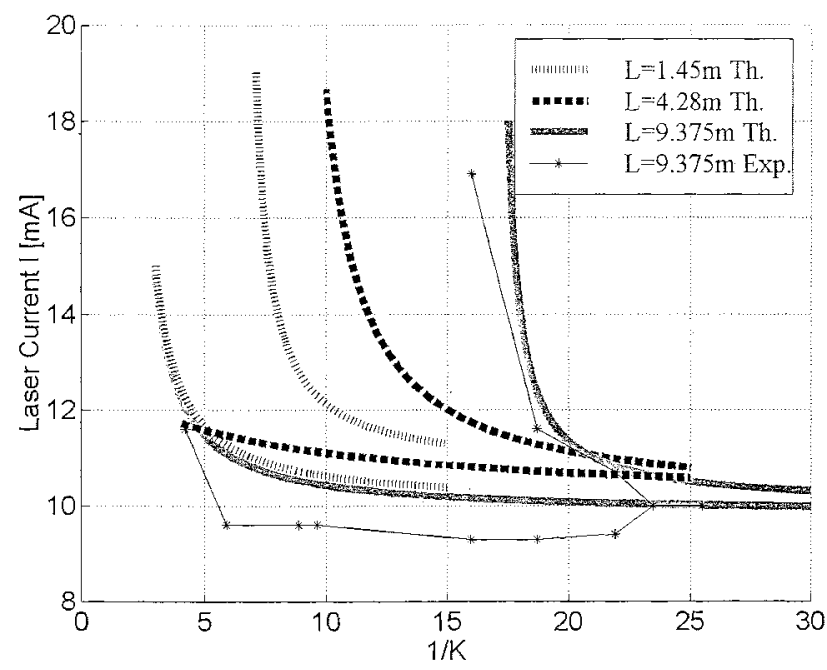

(b)

Fig. 4. (a) Boundaries of the chaotic regime: experimental points are reported for different lengths $L$ of the fiber cavity. The theoretical curve for $L=9.375 \mathrm{~m}$ is also shown for comparison. The spectra corresponding to points A, B, C, D are shown in Fig. 5. (b) Boundaries of the chaotic regime: theoretical curves are reported for different lengths $L$ of the fiber cavity. The experimental points for $L=9.375 \mathrm{~m}$ are also shown for comparison.

Experimental data have been obtained after polarization compensation by observing the polarization state at the mirror end. In the figures, they have been connected by a line for better reading. Some interesting experimental spectra corresponding to specific points of Fig. 4(a) are shown in Fig. 5.

Theoretical curves are based on (4) and(5). The numerical integration has been carried out by a standard fourth-order Runge-Kutta algorithm. We have assumed $\lambda=1551 \mathrm{~nm}$ for the solitary laser emission wavelength and $n_{b}=3.45$ for the refraction index of the bulk semiconductor material.

The remaining parameters $\left(\alpha, \tau_{p}, \tau_{s}, \tau_{\text {in }}, N_{t}, \xi\right)$ were numerically fitted, starting from typical trial values [4]. For $\alpha$, we obtained a rather large value $(\alpha \approx 6)$, which is perhaps due to the specific fabrication technology [DFB facet selective-growth buried heterostructure (DFB-FSBH)].

By this approach, we have been able to get a good agreement between theoretical and experimental data for three of the four cavity lengths, as shown in Fig. 4(a) and (b). (a)

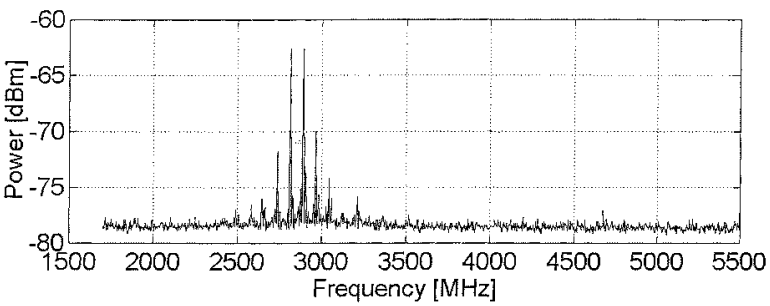

(b)

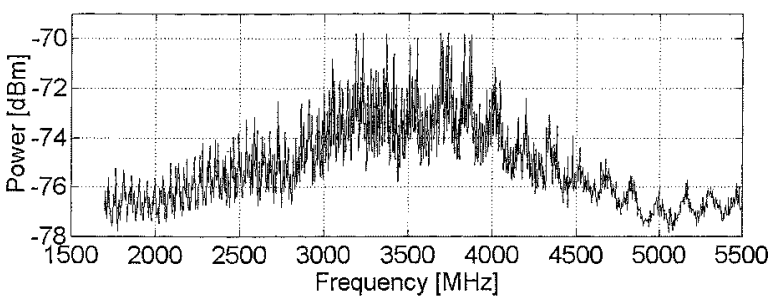

(c)
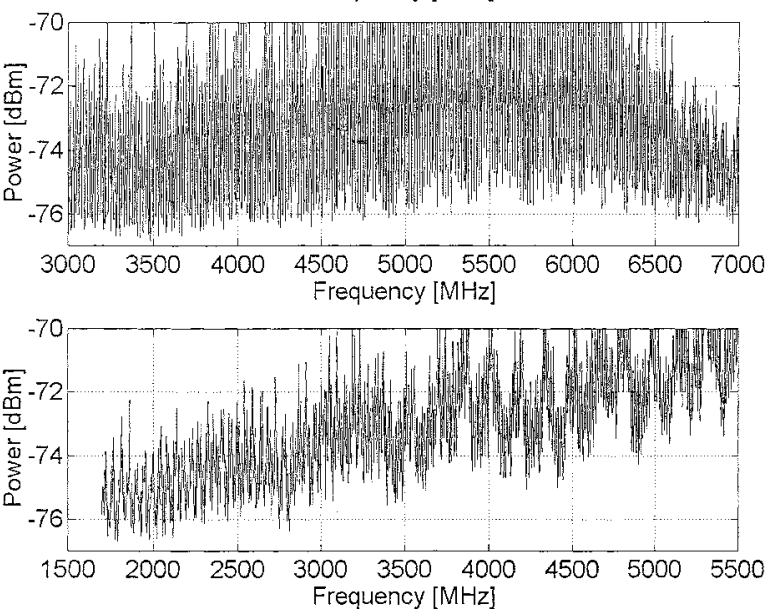

Fig. 5. Different spectra taken in different points (A,B,C,D) of Fig. 4(a).

However, quite surprisingly, the experimental curve for $L=$ $87.5 \mathrm{~cm}$ was found to lie on the left of that for $L=1.45 \mathrm{~m}$, in contrast with theory. This experimental observation has been confirmed by repeated measurements, and this behavior is being investigated in more detail.

From Fig. 4(a), it can be also observed that the experimental $K$ value corresponding to the onset of the chaotic region $K_{o}$ depends on $L$. However, since the laser works in the long-cavity regime, the value of $K$ corresponding to the onset of instabilities $K_{c}$, (detected by observation of enlarged linewidth, and an increase in the RIN), should not depend on $L$ [2]. As a check, we have experimentally observed the onset of instability for all four cavity lengths, finding that it actually comes approximately the same value $K_{c}=1.810^{-2}<K_{o}$.

From Fig. 4(a), it is evident that a longer cavity provides chaos for a larger range of current and for a lower $K$. More important, a long cavity is also advantageous since it gives a larger chaotic dimension, which is an important parameter to be considered in view of cryptographic applications.

This is a consequence of the laser with feedback being a differential delay system. As reported in [13], the dimension of the attractor, (which is approximately given by the positive Lyapunov exponents), and thus the complexity of chaos, is determined by the number of involved ECMs, which increases with the feedback level $K$ for a given delay $\tau$ (or length $L$ ). On the other side, the number of ECMs increases with $L$ for a given 


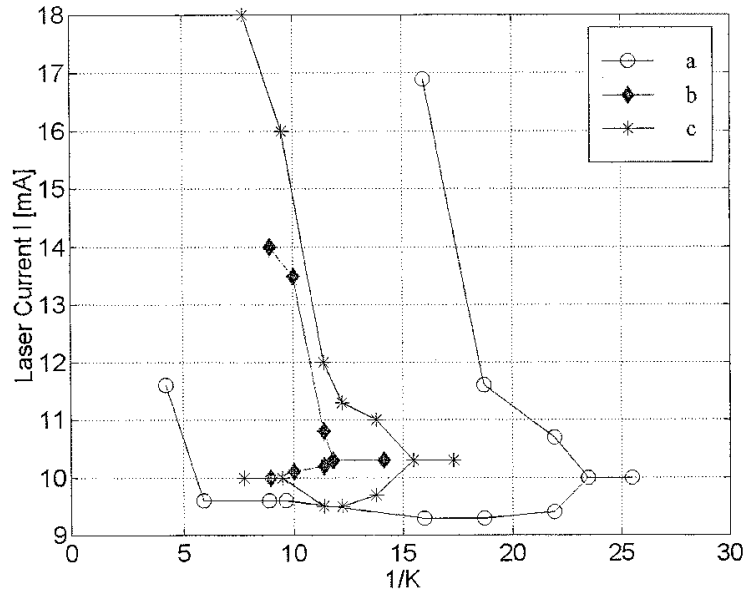

Fig. 6. Boundaries of the chaotic regime for different polarization states measured at the mirror end and $L=9.375 \mathrm{~m}$ (see text).

$K$. Therefore a high-dimension chaotic attractor, suitable for chaotic encryption, is achieved in a long-cavity simply because the system can explore (at a given feedback level $K$ ) a larger number of ECMs.

Thus, in our experiments, the chaos dimension has been estimated by counting the number $D$ of ECMs involved in the attractor, i.e., the peaks superimposed on the large continuous chaotic spectrum. Indeed, for the four different fiber lengths, the chaos dimension has been estimated to be $D=8,30,80$, and 130 from the shorter to the longer cavity, respectively. The increase in the chaos dimension with $L$ is also evident from comparison of Fig. 3 with Fig. 5.

The dependence of $K$ on the polarization state has been also investigated experimentally. In Fig. 6, the boundaries of the chaotic regime are shown for the longest cavity $(L=9.375 \mathrm{~m})$ considering three different polarization states: curve a has been obtained after polarization control, and is the same as shown in Fig. 4 for the same length; curve b corresponds to a linear polarization at $80^{\circ}$ with respect to that of the laser; and curve c corresponds to an intermediate elliptical polarization state. It is clear from Fig. 6 that polarization rotation, and/or ellipticity, results in a reduction of $K$, and that trimming the fiber birefringence provides a means to select the laser regime.

However, it must be observed that when the polarization of the feedback light does not coincide with that of the laser output, part of the injected light acts incoherently. Though this effect can be safely neglected in laser normal operation [24], where the laser capability for rejecting the rotated components has been used to implement an isolator, in the chaotic regime such components can influence the laser dynamics [25]. For this reason, trimming of $K$ by acting on the fiber birefringence is not completely equivalent to the trimming performed by a variable attenuator, unless a polarizer is put in front of the laser.

This observation is important in view of synchronization experiments, where one wants not only to get a chaotic spectrum of a given width and shape, but also to obtain the same time evolution from the outputs of two coupled chaotic lasers [8]-[12]. In this case, the use of a true attenuator is required. It can be provided, for example, by a fiber loop of suitable radius in the same plane of the fiber. The fiber should be carefully positioned

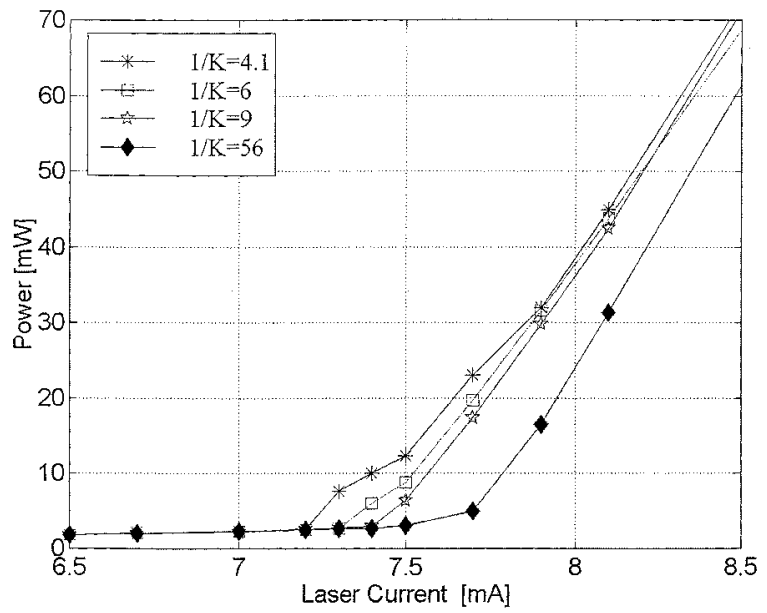

Fig. 7. $P$ versus $I$ curves for different $K$ values $(L=9.375 \mathrm{~m})$.

as explained in Section II. Moreover, fiber polarization has to be compensated for in the feedback path between the two lasers by one or more retarding loops, whose orientation has to be optimized within a few degrees.

Finally, in Fig. 7, we show a collection of $P$ versus $I$ diagrams measured on our laser for different values of $K$ to put into evidence the threshold variation. Such curves were measured for $L=9.375 \mathrm{~m}$.

\section{CONCLUSION}

In this paper, it has been shown that a standard telecommunications laser in the third window, subject to backreflection, exhibits a chaotic regime which is promising for hiding information in cryptographic applications. Such a regime arises at moderate values of reflection, which can be easily obtained in a fiber-optic setup. Cavity lengths of the order of a few meters can be implemented in a stable and compact way by a fiber-optic path, achieving a high dimension chaotic spectrum. Working with a standard telecommunications laser and a standard single-mode fiber represents the first step for the implementation of a chaotic transmitter-receiver system which will be easy to connect in the future to a standard fiber optic link.

\section{ACKNOWLEDGMENT}

The authors would like to acknowledge Prof. S. Donati for his continuous scientific support and A. Bruno for his help in the experimental activity.

\section{REFERENCES}

[1] R. Lang and K. Kobayashi, "External optical feedback effects on semiconductor injection laser properties," IEEE J. Quantum Electron., vol. QE-16, pp. 347-355, Mar. 1980.

[2] K. Petermann, "External optical feedback phenomena in semiconductor lasers," IEEE J. Select. Topics Quantum Electron., vol. 1, pp. 480-489, June 1995.

[3] J. Sacher, W. Erlsasser, and E. O. Gobel, "Nonlinear dynamics of semiconductor laser emission under variable feedback conditions," IEEE J. Quantum Electron., vol. 27, pp. 373-379, Mar. 1991.

[4] V. Annovazzi-Lodi, A. Scirè, M. Sorel, and S. Donati, "Dynamic behavior and locking of a semiconductor laser subjected to external injection," IEEE J. Quantum Electron., vol. 34, pp. 2350-2357, Dec. 1998. 
[5] D. Pieroux, T. Erneux, B. Haegeman, K. Engelborghs, and D. Roose, "Bridges of periodic solutions and tori in semiconductor lasers subject to delay," Phys. Rev. Lett., vol. 87, no. 19, pp. 1-4, Nov. 2001.

[6] J. Mork, B. Tromborg, and P. L. Christiansen, "Bistability and lowfrequenct fluctuations in semiconductor lasers with optical feedback," IEEE J. Quantum Electron., vol. 24, pp. 123-133, Feb. 1988.

[7] G. H. M. van Tartwijk, A. M. Levine, and D. Lenstra, "Sisyphus effect in semiconductor lasers with optical feedback," IEEE J. Select. Topics Quantum Electron., vol. 1, pp. 466-472, June 1995.

[8] V. Annovazzi-Lodi, S. Donati, and A. Scirè, "Synchronization of chaotic lasers by optical feedback for cryptographic applications," IEEE J. Quantum Electron., vol. 33, pp. 1449-1454, Sept. 1997.

[9] — - "Synchronization of chaotic injected-laser systems and its application to optical cryptography," IEEE J. Quantum Electron., vol. 32, pp. 953-959, June 1996.

[10] C. Mirasso, P. Colet, and P. Garcia-Fernandez, "Synchronization of chaotic semiconductor lasers: Application to encoded communications," IEEE Photon. Technol. Lett., vol. 8, pp. 299-301, Feb. 1996.

[11] S. Sivaprakasam and K. A. Shore, "Message encoding and decoding using chaotic external-cavity diode lasers," IEEE J. Quantum Electron., vol. 36, pp. 35-39, Jan. 2000.

[12] J.-B. Cuenot, L. Larger, J.-P. Goedgebuer, and W. T. Rhodes, "Chaos shift keying with an optoelectronic encryption system using chaos in wavelength," IEEE J. Quantum Electron., vol. 37, pp. 849-855, July 2001.

[13] C. Masoller, "Coexistence of attractors in laser diode with optical feedback from a large external cavity," Phys. Rev. A, vol. 50, no. 9, pp. 2569-2578, Sept. 1994.

[14] J. Mork, B. Tromborg, and J. Mark, "Chaos in semiconductor lasers with optical feedback: Theory and experiment," IEEE J. Quantum Electron., vol. 28, pp. 93-107, Jan. 1992.

[15] T. Heil, I. Fisher, W. Erlsasser, J. Mulet, and C. R. Mirasso, "Statistical properties of low-frequency fluctuations during single-mode operation in distributed-feedback lasers; Experiments and modeling," Opt. Lett., vol. 24 , no. 18 , pp. 1275-1277, Sept. 1999.

[16] R. Ulrich, "Polarization stabilization on single mode fibers," Appl. Phys. Lett., vol. 35, no. 11, pp. 840-843, 1979.

[17] R. L. Davichack, Y. C. Lai, A. Gavrielides, and V. Kovanis, "Chaotic transitions and low-frequency fluctuations in semiconductor laser with optical feedback," Physica D, vol. 145, pp. 130-143, 2000.

[18] P. M. Alsing, V. Kovanis, and A. Gavrielides, "Lang and Kobayashi phase equation," Phys. Rev. A, vol. 53, no. 6, pp. 4429-4434, June 1996.

[19] B. Tromborg, J. H. Osmundsen, and H. Olesen, "Stability analysis for a semiconductor laser in an external cavity," IEEE J. Quantum Electron., vol. QE-20, pp. 1023-1033, Sept. 1984.

[20] R. W. Tkach and A. R. Chraplyvy, "Regimes of feedback effects in 1.5 $\mu \mathrm{m}$ distributed feedaback lasers," J. Lightwave Technol., vol. LT-4, pp. 1655-1661, Nov. 1986.

[21] T. Heil, I. Fisher, and W. Erlsasser, "Influence of amplitude-phase coupling in the dynamics of semiconductor lasers subject to optical feedback," Phys. Rev. A, vol. 60, no. 1, pp. 634-641, July 1999.

[22] R. J. Jones, P. S. Spencer, J. Lawrence, and D. M. Kane, "Influence of the external cavity length on the coherence collapse regime in laser diodes subject to optical feedback," Proc. IEE-Optoelectronics, vol. 148, no. 1, pp. 7-12, Feb. 2001

[23] T. Heil, I. Fisher, W. Erlsasser, and A. Gavrielides, "Dynamics of semiconductor lasers subject to delayed optical feedback: The short cavity regime," Phys. Rev. Lett., vol. 87, no. 24, pp. 243 901-1-243 901-4, Dec. 2001.

[24] V. Annovazzi Lodi, S. Donati, S. Merlo, L. Zucchelli, and F. Martinez, "Protecting a pump laser-diode from retroreflections by means of a fiber $\lambda / 4$ retarder," IEEE Photon. Technol. Lett., vol. 8, pp. 485-487, Apr. 1996.

[25] J. Houdian, G. Huyet, and J. G. McInerney, "Dynamics of a semiconductor laser with incoherent optical feedback," Opt. Commun., vol. 199, pp. 175-179, Nov. 2001.
Valerio Annovazzi-Lodi (M'89-SM'99) was born in Novara, Italy, in 1955. He received the degree in electronic engineering from the University of Pavia, Pavia, Italy, in 1979.

Since then, he has been with the Department of Electronics, University of Pavia, working in the fields of electronics and electrooptics. In 1983, he became a Staff Researcher in the Department of Electronics, in 1992 an Associate Professor, and in 2001 a Full Professor. His main research interests include injection phenomena and chaos in oscillators and lasers, electrical fiber sensors, fiber gyroscopes, passive fiber components for telecommunications and sensing, optical amplifiers, transmission via diffused infrared radiation, and micromechanical systems. He is the author of more than 80 papers and holds four patents.

Dr. Annovazzi-Lodi is a member of the Associazione Elettrotecnica Italiana (AEI)

Sabina Merlo (M'01) was born in Pavia, Italy, in 1962. She received the degree in electronic engineering from the University of Pavia, Pavia, Italy, in 1987, the M.S.E. degree in bioengineering from the University of Washington, Seattle, in 1989, and the Ph.D. degree in electronic engineering from the University of Pavia in 1991.

In 1993, she became an Assistant Professor, and in 2001 an Associate Professor with the Department of Electronics, University of Pavia. Her main research interests include fiber-optic passive components and sensors, optical amplifiers, chaos in lasers, laser interferometry, and microelectromechanical systems. She holds three patents and is the author of approximately 50 papers.

Dr. Merlo was the recipient of a Rotary Foundation Graduate Scholarship to study at the University of Washington. She is a member of the Associazione Elettrotecnica Italiana (AEI)

Michele Norgia (S'98-M'00) was born in Omegna, Italy, in 1972. He received the degree in electronics engineering in 1996, with a thesis on noise in optical amplifiers, and the Ph.D. degree in electronics engineering and computer science in 2000, both from the University of Pavia, Pavia, Italy

In 1997, he spent five months as visiting student at C.S.E.L.T., Torino, Italy, where he worked on fiber Bragg gratings. He is currently working in the Optoelectronics Group of the University of Pavia. His main research interests are noise in optical amplifiers, chaos in lasers, interferometry, and microelectromechanical sensors. He is the author of 20 papers.

Dr. Norgia is a member of the IEEE Lasers and Electro-Optics (LEOS) Society.

Alessandro Scirè (M'99) was born in Rimini, Italy, in 1971. He received the degree in electronic engineering and the Ph.D. degree in electronics engineering and computer science from the University of Pavia, Pavia, Italy, in 1995, working in the Optoelectronics Group.

$\mathrm{He}$ is presently with the Instituto Mediterraneo de Estudios Avanzados (IMEDEA), Mallorca, Spain, as a recipient of a Marie Curie Fellowship from the European Community. His research interests include modeling of laser diodes and of active optical components, dynamics of vertical-cavity surfaceemitting lasers (VCSELs) due to saturable absorption, VCSEL arrays, coupled vectorial oscillators, semiconductor ring lasers, and nonlinear dynamics in optically injected laser diodes. He is the author of 20 papers. 